\title{
Macroorchidisme et spermatogenèse dans le syndrome de l'X fragile
}

\author{
A. PAGES 1 , A. GENTIL-PERRET ${ }^{2}$, R. LEVY ${ }^{3}$, C. ARMAND ${ }^{1}$, J. TOSTAIN $^{1}$ \\ 1 Urologie-Andrologie ; 2 Anatomo-pathologie ; 3 Laboratoire de Biologie de la Reproduction, C.H.U. Hôpital \\ Nord, Saint-Etienne, FRANCE
}

\section{RÉSUMÉ}

Le syndrome de l'X fragile est lié à l'expansion d'une séquence répétitive de triplets CGG dans la région 5' du gène FMR1. II en résulte l'absence de production de la protéine FMRP par blocage de la transcription du gène FMR1. Nous rapportons une étude hormonale, histologique et immunohistochimique d'un cas, centrée sur les fonctions testiculaires et l'origine du macroorchidisme.

Le syndrome de l'X fragile s'accompagne d'une spermatogenèse normale ou incomplète ou encore présentant des lésions aux stades tardifs. Ces lésions tardives associées à l'œdème interstitiel s'apparentent aux lésions observées chez les vasectomisés ce qui a fait soulever l'hypothèse du rôle d'une hyperpression péritubulaire et/ou d'anticorps anti-spermatozoïdes. Dans notre observation, la négativité des anticorps anti-spermatozoïdes et la discrétion de l'œdème ne sont pas en faveur de cette hypothèse. L'histologie retrouve l'existence d'une hyperplasie du tissu interstitiel, d'un épaississement de la membrane basale péritubulaire et d'une augmentation du diamètre externe des tubes séminifères sans anomalie morphologique des cellules de Leydig ou des cellules de Sertoli.

Les immunomarquages réalisés montrent la présence de la protéine FMRp dans les cellules germinales ce qui indique que ces cellules ne sont pas porteuse de la mutation complète. Cette expression de la protéine FMRp dans le testicule peut permettre d'expliquer la conservation de la spermatogenèse alors même qu'il a été suggéré que la protéine FMRp soit nécessaire la survie des cellules germinales. Nos résultats sont compatible avec l'hypothèse que les atteintes différentes de la spermatogenèse selon les patients atteints du syndrome puissent être liées au nombre de cellules germi- nales touchées par la mutation complète. Des travaux complémentaires sont toutefois nécessaires pour comprendre la variabilité des atteintes de la spermatogenèse et la physiopathologie du macroorchidisme dans le syndrome de l'X fragile.

Mots clés :Fertilité, Macroorchidisme, Spermatogenèse, Syndrome de I'X Fragile

\section{INTRODUCTION}

Le syndrome de l'X fragile, ou syndrome de Martin-Bell, a été décrit pour la première fois en 1909. Cette affection, liée au chromosome $X$ est, dans la majorité des cas, entrâ̂née par l'expansion d'une séquence répétitive de triplets CGG dans la région 5' du gêne FMR1 (Fragile X Mental Retardation 1 gene) [25]. Alors que le nombre de triplet est normalement entre 6 et 50, une augmentation du nombre de triplet entre 50 et 200 caractérise un état dit de « prémutation » qui ne provoque pas d'atteinte clinique mais qui peut, lorsqu'il est présent chez une femme, se déstabiliser à la génération suivante pour donner chez l'enfant une augmentation du nombre de triplet dépassant 200 et pouvant atteindre 2000, situation que l'on qualifie de « mutation complète ». Au delà de 230 triplets, une méthylation anormale de l'ADN de cette région bloque la transcription du gène FMR1, empêchant ainsi la production de la protéine FMRP (Fragile X Mental Retardation Protein). Cette répression du gène FMR1 serait responsable du phénotype de l'X fragile $[22,24]$.

\section{Correspondance :}

Dr. Arnaud PAGES, Urologie-Andrologie, C.H.U. Hôpital Nord, Avenue Albert Raimond, 42055 Saint-Etienne Cedex 2, France 
Sa prévalence est de 1 cas sur 1500 naissances pour les garçons et de 1 cas sur 2500 naissances pour les filles. A la différence d'autres affections génétiques fréquentes, ce syndrome s'observe dans tous les groupes ethniques et peut donc être considéré comme l'une des pathologies humaines les plus fréquentes liées à un seul gène $[12,22]$.

Le diagnostic clinique repose sur l'association d'un retard mental et d'une dysmorphie faciale évocatrice. Dans le sexe masculin, un macroorchidisme post-pubertaire supérieur à $30 \mathrm{ml}[5,8,9,10,13]$ est observé dans $80 \%$ des cas. Le diagnostic biologique fait maintenant appel à des techniques d'hybridation (Southern Blot) ou d'amplification génique (PCR) [6].

Nous rapportons une étude hormonale, histologique et immuno-histochimique permettant de discuter l'origine du macroorchidisme et la variabilité des fonctions testiculaire des patients porteurs de la mutation.

\section{MATERIEL ET METHODES}

Après information et consentement éclairé, des prélèvements sanguins pour étude génétique et hormonale ainsi que des biopsies testiculaires bilatérales ont été effectuées lors d'une intervention chirurgicale pour cure d'hydrocèle chez un jeune homme de 21 ans, deuxième enfant d'une fratrie de deux, présentant la triade clinique typique du syndrome de l'X fragile. Le père et le frère n'étaient pas porteurs de la mutation, alors que la mère présentait la prémutation.

L'étude de la fragilité du chromosome $X$ fut réalisée sur sang périphérique par les méthodes moléculaires évaluant la taille du site FRAXA (Southern blot avec la sonde St B12.3). Des dosages hormonaux de testostérone, FSH et LH ont été effectués par méthode immunologique. Une recherche d'anticorps anti-spermatozoïdes circulants sériques a été réalisée par une méthode d'agglutination.

Pour l'étude histologique, les prélèvements testiculaires fixés dans du liquide de Bouin ont été inclus en paraffine avant section en coupes de $5 \mu \mathrm{m}$ d'épaisseur et coloration par l'hémalun-phloxine-safran. L'étude immuno-histochimique a été pratiquée sur coupes déparaffinées à l'aide d'anticorps primaires monoclonaux dirigés contre l'antigène nucléaire Ki-67 (clone MIB-1; 1/50, Immunotech Marseille, France) et contre la protéine FMRP (Ac antiFMRP clone $1 \mathrm{C} 3 \mathrm{la}, 1 / 50$, societé Euromedex) en utilisant la technique d'immunoperoxydase indirecte en 3 couches [18]. Brièvement, les coupes déparaffinées ont été réhydratées, incubées 40 minutes avec l'anticorps primaire, puis 30 minutes avec un anticorps secondaire lapin antisouris marqué à la peroxydase $(1 / 20$, polyclonal Dako,Glostrup, Danemark), enfin 30 minutes avec un anticorps tertiaire porc anti-lapin également marqué à la per- oxydase (1/20, polyclonal Dako,Glostrup, Danemark). La réaction immunologique a été révélée par le chromogène diaminobenzidine 3-3 (Sigma, Saint Louis, Missouri). Les lames ont été contre-colorées par l'hématoxilline de Harris. Les cellules marquées positives étaient ainsi colorées en marron. Un contrôle négatif a été effectué en omettant l'incubation avec l'anticorps primaire. Des lames de parenchyme testiculaire normal ont été, par ailleurs, utilisées comme témoins.

\section{RESULTATS}

L'échographie pré-opératoire retrouvait un volume testiculaire moyen de $36 \mathrm{ml}$.

Sur le plan biologique, les dosages hormonaux indiquaient des valeurs normales de Testostérone totale à $661 \mathrm{ng} /$ $100 \mathrm{ml}$ ( $\mathrm{N}: 300-1200)$, et de LH à 2 UI/L (N :1-8). La FSH était inférieure à la normale à $0.4 \mathrm{UI} / \mathrm{L}(\mathrm{N}: 1,2-11)$. La recherche d'Anticorps anti-spermatozoïdes (ACAS) sériques était négative.

La lecture en microscopie optique a permis de visualiser un tissu interstitiel congestif, parfois œedématié, comportant des îlots leydigiens en nombre normal. Les tubes séminifères avaient pour la plupart un diamètre satisfaisant (diamètre moyen $265 \mu \mathrm{m}$; extrêmes: $253-288 \mu \mathrm{m}$ ), et $10 \%$ seulement étaient atrophiques et scléro-hyalins (Figure 1). Les cellules de Sertoli étaient en nombre normal. Les tubes comportant une lumière contenaient une lignée germinale de densité et de maturation subnormales aboutissant à la production d'assez nombreuses formes allongées, spermatides et spermatozoïdes qui s'éliminaient dans une lumière sous forme libre ou nécrobiotique (Figure 2). La membrane péritubulaire était subnormale ou le siège d'un épaississement fibreux très discret. Les biopsies testiculaires furent classées T2-M1-I0 selon la classification de Valette [27].

En immunohistochimie, on notait une hyper expression de la protéine FMRp au sein du cytoplasme des cellules de Sertoli et des cellules de Leydig. Il existait une expression cytoplasmique avec renforcement membranaire au sein des cellules germinales jusqu'au stade de spermatide. Les lames témoins comparatives donnaient des résultats superposables (Figure 3).

\section{DISCUSSION}

Aucune des hypothèses sur l'origine de la macroorchidie n'est unanimement admise. Elle peut être présente en dehors de toute anomalie génétique [17] et dans une autre affection liée à l'X sans mutation du gêne FMR1 [14]. Chez le garçon atteint d'X fragile, le volume testiculaire est significativement plus important que chez les garçons non atteints dès l'âge de 3 ans, mais le macroorchidisme vrai ( $\geq 2$ fois la normale) se développerait habituellement entre 6 et 8 ans [13]. En fait, cette anomalie a été rapportée dès 


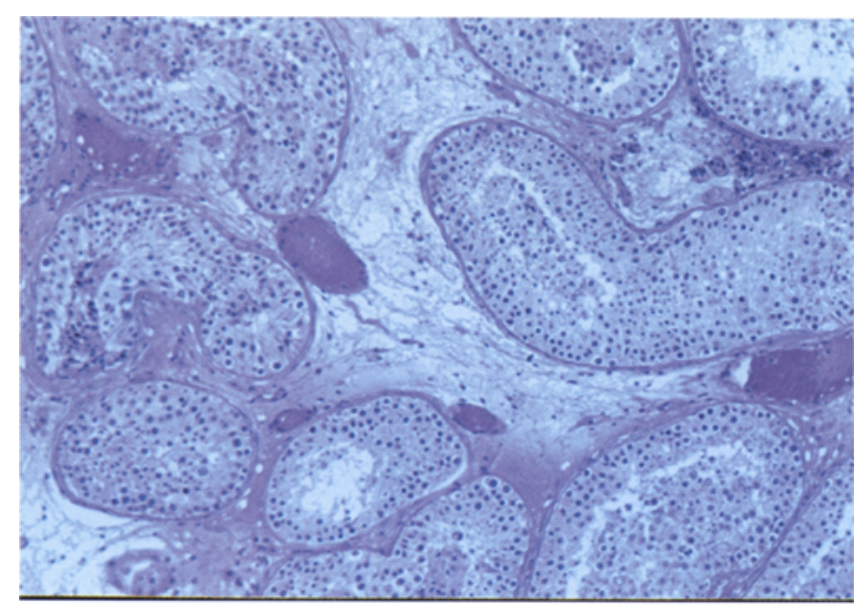

Figure 1 : Préparation histologique (Hemalun-Phloxine-Safran; grossissement original $x 40$ ) mettant en évidence un odème interstitiel et une congestion vasculaire. Les tubes séminifères ont un diamètre régulier.

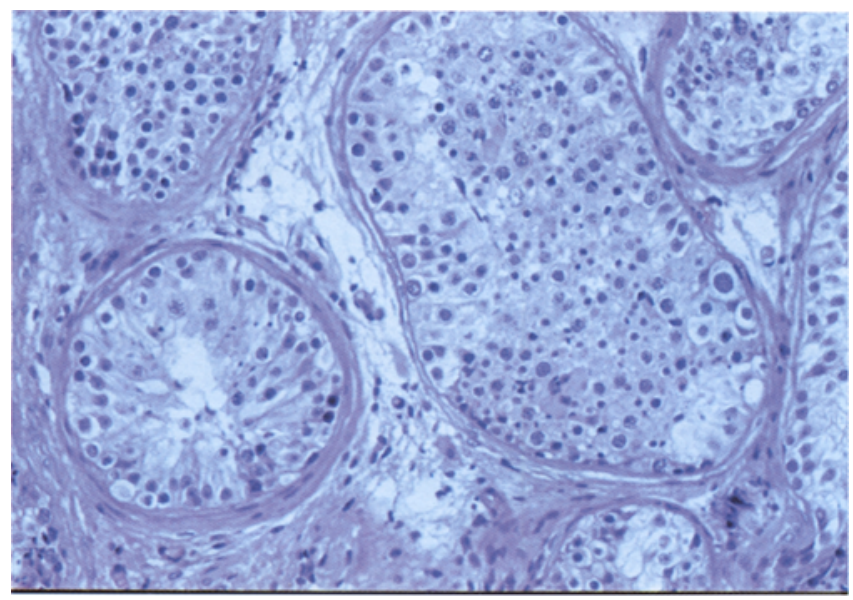

Figure 2: Préparation histologique (Hemalun- Phloxine- Safran; grossissement original $x 10)$ montrant un épaississement discret et inconstant de la membrane péri-tubulaire. La spermatogenèse aboutit à la formation d'assez nombreux spermatides et spermatozoüdes.

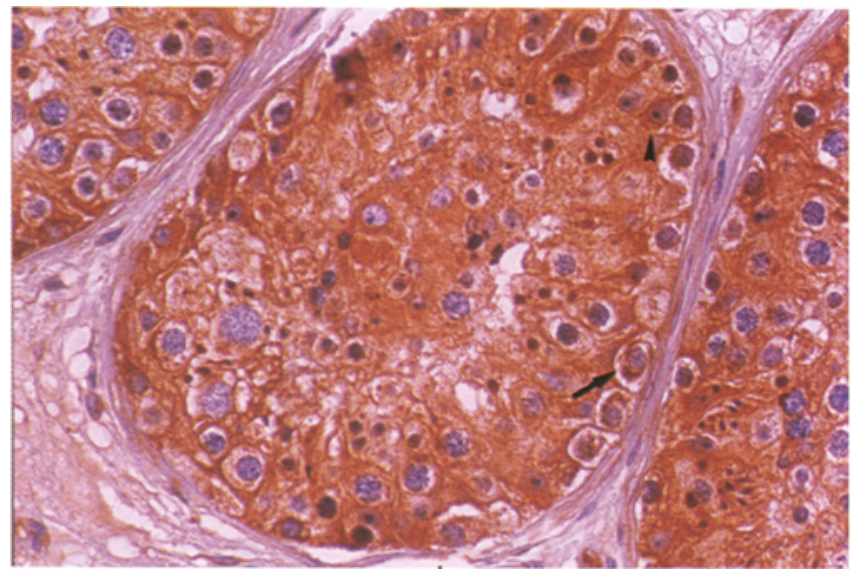

Figure 3: Préparation histologique (Immunomarquage à l'aide de l'anticorps antiFMRp). L'expression de la protéine FMRp par les cellules germinales (flèches) et les cellules de Sertoli (têtes de flèches l'âge de 5 mois [5]. Il est donc probable que la rareté des observations de macroorchidisme chez le très jeune enfant est liée à l'absence de mesure systématique. Le parenchyme testiculaire ne présente aucune anomalie échographique [9]. Le macroorchidisme n'est pas lié à un trouble hormonal hypothalamo-hypophyso-gonadique [20]. Turner en 1975 [26], relayé par d'autres auteurs [3, 4, 11], a décrit une hyperplasie du tissu interstitiel et des cellules de Leydig ainsi qu'un épaississement de la membrane basale péritubulaire. Dans des modèles animaux, une prolifération anormale des cellules de Sertoli, limitée à la période embryonnaire et la période néonatale, a été rapportée indépendamment de toute anomalie du signal de transduction de la FSH [12]. En microscopie électronique, on a observé un oedème interstitiel avec une augmentation du diamètre externe et de la longueur des tubes séminifères au détriment du diamètre luminal $[17,20]$. Une hyperplasie des cellules de Sertoli, qui étaient en nombre normal mais présentaient parfois des anomalies à type de cytoplasme granuleux et de noyau d'aspect immature, a été rapportée $[11,20]$. L'augmentation de longueur et de diamètre des tubules expliquerait l'augmentation de volume testiculaire.

Dans notre observation, les mesures du diamètre tubulaire donnent effectivement des résultats supérieurs aux valeurs habituellement admises de $200 \pm 20 \mu \mathrm{m}$ [20], permettant d'expliquer en partie la macroorchidie. Par contre, comme d'autres auteurs l'ont déjà noté $[17,20]$, l'œdème interstitiel était trop discret pour jouer un rôle significatif. Certains ont émis l'hypothèse d'une sensibilité exacerbée des tubes séminifères à la FSH [17]. Dans notre observation les taux de FSH étaient anormalement bas, mais dans la littérature ils apparaissent normaux [3] et Nistal a même rapporté une augmentation modérée de la FSH [20]. Nous n'avons pas observé d'augmentation du nombre de cellules de Sertoli comme cela a été décrit chez la souris dont le gène FMR1 a été inactivé [12].

La spermatogenèse des patients atteints du syndrome de $1 \mathrm{X}$ fragile est décrite soit comme normale $[3,4,26$, notre observation], tantôt incomplète, réduite à la présence de spermatogonies, spermatocytes et de rares spermatides [20]. Pour d'autres, la spermatogenèse initiale serait normale, mais laisserait apparaître des lésions tardives au niveau des spermatides $[11,13,24]$. Ces lésions germinales et les lésions histologiques décrites présenteraient des similitudes avec celles observées chez les vasectomisés [11], permettant aux auteurs de soulever les hypothèses d'une hyper-pression par obstacle péritubulaire lié à l'œdème interstitiel et/ou de la présence d'anticorps anti-spermatozoïdes. La discrétion de l'œdème et la négativité des recherches d'anticorps dans notre observation ne vont pas dans ce sens. Le rôle de FMRP, qui est localisée dans le cytoplasme [7], n'est pas encore élucidé. Reyniers la considérait comme indispensable à la survie des gamètes [23], 
hypothèse réfutée par Bakker qui n'observait pas d'anomalie sur un modèle expérimental [2]. Bachner, après une étude en immunomarquage montrant une hyperexpression de FMRP au sein des spermatogonies de fotus de souris, déclinant rapidement à l'âge adulte, supposait que celle-ci était utile à la multiplication des cellules germinales [1]. Dans les épithéliums, l'expression de la FMRP est effectivement plus élevée dans les couches en division [10]. L'expression du gêne FMRl semblerait plus marquée dans la période foetale et infantile, assez ubiquitaire avec une transcription plus importante dans le cerveau, le cervelet et le testicule $[9,14]$. En fait, deux protéines homologues à la FMRP ont été décrites: FXR1 et FXR2. Ces protéines auraient des interactions entre elles et avec FMRP [24]. L'expression de ces trois protéines dans le testicule fotal et adulte suggère des fonctions indépendantes pour chacune d'entre elles durant le développement embryonnaire et la vie adulte [25]. A l'âge adulte, FMRP n'existerait, au sein du testicule, que dans les spermatogonies [7]. Chez notre patient, la spermatogénese est normale et les immunomarquages ne montrent aucune carence en FMRP dans les cellules testiculaires jusqu'aux spermatides. Des observations de transmission familiale dans la population mâle semblent également indiquer que la FMRP n'est pas indispensable à la spermatogenèse [19]. On sait que l'expansion de triplets n'est pas identique d'un patient à l'autre et, chez un même patient, d'un tissu à l'autre et à l'intérieur même d'un unique tissu [6]. Il est donc probable, par analogie avec des mosaïques humaines où le degré de méthylation de l'ADN est variable, que l'anomalie du gêne FMR1 présente des degrés autorisant une certaine transcription marquée par des atteintes moins sévères et, pour certains patients, une spermatogenèse compatible avec une descendance. L'opinion communément admise est que le sperme des patients porteurs d'un $\mathrm{X}$ fragile ne serait porteur que de la prémutation $[1,8,22]$. La transmission à la descendance ne pourrait alors se faire que sous forme de prémutation répondant à deux hypothèses compatibles avec l'expression de FMR1 dans les cellules germinales souches [1]:

Les spermatogonies porteuses de la mutation complète ne pourraient atteindre le stade de la méiose (théorie de la sélection).

Le passage de la prémutation allélique à la mutation complète ne s'effectuerait qu'après individualisation de la lignée germinale et serait limitée aux cellules somatiques (théorie de la restriction). Reyniers évoque ainsi la possibilité d'une expansion post-zygotic de la séquence de triplets. En fait, la mise en évidence de l'expansion de triplets évoquant la mutation complète dans des ovocytes de foetus et la mutation incomplète dans des testicules fœtaux rend cette hypothèse moins probable [16].

\section{CONCLUSION}

Le macroorchidisme observé dans le syndrome de l'X fragile ne paraît pas expliqué par l'cè̀me interstitiel, mais plutôt par l'augmentation du diamètre et de la longueur des tubes séminifères. L'origine de cette anomalie reste inconnue.

Nos constations confirment que la spermatogenèse de tels patients peut-être quasi-normale. L'existence d'observations d'individus stériles renforce la théorie de la sélection et de la probabilité d'une expression hétérogène de l'anomalie du gène FMR1.

\section{RÉFÉRENCES}

1. BACHNER D., MANCA A., STEINBACH P., WOHRLE D., JUST W., VOGEL W., HAMEISTER H., POUSTKA A. : Enhanced expression of the murine FMR 1 gene during germ cell proliferation suggests a special function in both the male and the female gonad. Hum. Mol. Genet., 1993, 2 : 2043-2050.

2. BAKKER C.E., VERHEIJ C., WILLEMSEN R., VAN DER HELM R., OERLEMANS R. : Fmr- 1 Knockout Mice: a model to study fragile $\mathrm{X}$ mental retardation. Cell, 1994, $78: 23-33$.

3. BERKOVITZ G.D., WILSON D.P., CARPENTER N.J., BROWN T.R., MIGEON C.J. : Gonadal function in men with the Martin-Bell (fragile-X) syndrome. Am. J. Med. Genet., 1986. 23 : 227-239.

4. BOWEN P., BIEDERMAN B., SWALLOW K.A. : The $X$ linked syndrome of macroorchidism and mental retardation: further observations Am. J. Med. Genet. 1978; 2 : 409-414.

5. CARMI R., MERYASH D.L., WOOD J., GERALD P.S. : Fragile-X syndrome asceratined by the presence of macro-orchidism in a 5-month-old infant. Pediatrics, 1984, $74: 883-886$.

6. DE GRAAFF E., WILLEMSEN R., ZHONG N., DE DIESMULDERS C.E., BROWN W.T., FRELING G., OOSTRA B. : Instability of the CGG repeat and expression of the FMR 1 protein in a male fragile $X$ patient with a lung tumor. Am. J. Hum. Genet., 1995, $57: 609-618$.

7. DEVYS D., LUTZ Y., ROUYER N., BELLOCQ J.P., MANDEL J.L. : The FMR-1 protein is cytoplasmic, most abundant in neurons and appears normal in carriers of a fragile $\mathrm{X}$ premutation. Nature Genet., 1993, $4: 335-340$.

8. HINDS H.L., ASHLEY C.T., SUTCLIFFE J.S., NELSON D.L., WARREN S.T., HOUSMAN D.E., SCHALLING M. : Tissue specific expression of FMR-1 provides evidence for a functional role in fragile X syndrome. Nature Genet., 1993, 3 : 36-43.

9. HUTTON L., RANKIN R.N., POSZONYI J. : High resolution ultrasound of macro-orchidism in mental retardation. J. Clin. Ultrasound., 1985, $13: 19-22$.

10. ITO M., SUGIE H. : Age dependent and tissue specific FMR-1 gene expression in human organs. Nippon Rinsho, 1999, 57 : 950954.

11. JOHANNISSON R., REHDER H., WENDT V., SCHWINGER E. : Spermatogenesis in two patients with the fragile $\mathrm{X}$ syndrome. I. Histology: light and electron microscopy. Hum. Genet., 1987, $76: 141-147$.

12. SLEGTENHORST-EEGDEMAN K.E., DE ROOIJ D.G., VERHOEF-POST M., VAN DE KANT H.J., BAKKER CE., 
OOSTRA B.A., GROOTEGOED J.A., THEMMEN A.P. : Macroorchidism in FMR1 knockout mice is caused by increased Sertoli cell proliferation during testicular development. Endocrinology, 1998, $139:$ 156-162.

13. LACHIEWICZ A.M., DAWSON D.V.: Do young boys with fragile $\mathrm{X}$ syndrome have macroorchidism?. Pediatrics, 1994, 93 : 992-995.

14. LINDSAY S., SPLITT M., EDNEY S., BERNEY T.P., KNIGHT S.J., DAVIES K.E., O’BRIEN O., GALE M., BURN J. : PPM-X: a new $\mathrm{X}$-linked mental retardation syndrome with psychosis, pyramidal signs, and macroorchidism maps to Xq28. Am. J. Hum. Genet., 1996, 58 : 1120-1126.

15. LUBS H.A. : A marker X chromosome. Am. J. Hum. Genet., $1969,21: 231$.

16. MALTER H.E., IBER J.C., WILLEMSEN R., DE GRAAF E., TARLETON J.C., LEISTI J., WARREN S.T., OOSTRA B.A. : Characterization of the full fragile $X$ syndrome mutation in fetal gametes. Nature Genet., 1997, $15:$ 165-169.

17. MARTINEZ-GARCIA F., REGADERA J., COBO P., MARTINCORDOVA C., PANIAGUA R., NISTAL M. : Macro-orchidism: new pathogenic and histopathologic aspects. Arch. Esp. Urol., 1994, $47:$ :59-65.

18. MASON D.Y., SAMMONS R.E. : The labeled antigen method of immunoenzymatic staining. J. Histochem. Cytochem.,1979, 14 : 291-302.

19. MEIJER H., DE GRAAF E., MERCKX D.M., JONGBLOED R.J., DE DIE-SMULDERS C.E., ENGELEN J.J., FRYNS J.P., CURFS P.M., OOSTRA B.A. : A deletion of $1.6 \mathrm{~kb}$ proximal to the CGG repeat of the FMR1 gene causes the clinical phenotype of the fragile X syndrome. Hum. Mol. Genet., 1994, 3 : 615-620.

20. NISTAL M., MARTINEZ-GARCIA F., REGADERA J., COBO P., PANIAGUA R. : Macro-orchidism: light and electron microscopic study of four cases. Hum. Pathol., 1992, $23: 1011-1018$.

21. OOSTRA B.A. : A fragile gene. Bioessays, 1995, $17: 941-947$.

22. PIERETTI M. : Abscence of expression of the Fmr-1 gene in fragile X syndrome. Cell, 1991, $66: 817-822$.

23. REYNIERS E., VITS L., DE BOULLE K., VAN ROY B. : The full mutation in the Fmr-1 gene of male fragile $X$ patients is absent in their sperm. Nature Genet., 1993, 4 : 143-146.

24. TAMANINI F., WILLEMSEN R., VAN UNEN L., BONTEKOE C., GALJAARD H., OOSTRA B.A., HOOGEVEN A.T. : Differential expression of FMR1, FXR1 and FXR2 proteins in human brain and testis. Hum. Mol. Genet., 1997, 6 : 1315-1322.

25. THOMAS.C. : Fragile X Syndrome. Improving understanding and diagnosis. J.A.M.A., 1994, $19: 7-21$.

26. TURNER G., EASTMAN C., CASEY J. : X-linked mental retardation associated with macroorchidism. Journal of medecine genetics. 1975, $12: 367-371$.

27. VALETTE J., GRIMAUD J.A., LANSAC J. : Essai d'interpretation standardisée histopathologique testiculaire dans le cadre de la fértilité masculine. Gynécologie, 1976, 27 : 219-223.

\begin{abstract}
Macroorchidism and spermatogenesis in fragile $X$ syndrome
\end{abstract}

\author{
A. PAGES, A. GENTIL-PERRET, R. LEVY, \\ C. ARMAND, J. TOSTAIN
}

Fragile $X$ syndrome is associated with expansion of a repeated triple CGG sequence in the 5' region of the FMR-1 gene, resulting in absence of production of FMRp protein due to blocking of the FMR1 transcription gene. The authors report a hormonal, histological, and immunohistochemical study of one case, focusing on the origin of macroorchidism and the variability of testicular functions in these patients.

Our observations confirm the existence of interstitial tissue hyperplasia, thickening of the peritubular basal membrane and an increased external diameter of the seminiferous tubes with no morphological anomalies in the Leydig or Sertoli cells.

Spermatogenesis in fragile $X$ Syndrome patients has been extensively studied: it may be normal, incomplete or patients may present impaired spermatogenesis in the late stages. These later lesions, which are associated with interstitial oedema, resemble the lesions observed after vasectomy, suggesting the role of raised peritubular pressure and anti-sperm antibodies. In our study, no antibodies were detected in the serum and oedema was minimal, which tends to discredit this theory.

The role of the FRMp protein in spermatogenesis remains controversial. Immunolabelling on slides prepared from testicular biopsies confirms that a complete form of mutation is not seen in the germ cells. Although the FMRp protein might be necessary for survival of germ cells, its persistent expression in the testis could explain preservation of spermatogenesis in some patients. Our results are consistent with the hypothesis that the varying degrees of impaired spermatogenesis could be related to the number of germ cells not affected by the complete mutation. However, additional data are necessary to fully understand the variability of spermatogenesis and the pathophysiology of macroorchidism observed in patients with fragile $X$ syndrome.

Keywords: Fertility, Fragile $X$ syndrome, Macroorchidism, Spermatogenesis. 